\title{
Une biologie de retardataires
}

\author{
Jacques Ninio
}

D 1 1 y a parfois des choses à glaner dans une pile de données. Ainsi, une exploitation systématique de la formidable base de données que constitue l'ensemble des annuaires téléphoniques de France pourrait faire apparaître quelques corrélations significatives, par exemple entre le sexe d'un abonné et le caractère pair ou impair des chiffres qui composent son numéro de téléphone. Peut-être pourrions-nous initier de telles recherches, en France, pour affiner nos outils conceptuels, en préambule à un engagement massif dans le programme du génome humain. La principale utilité de celui-ci, ainsi que l'a souligné Walter Gilbert [1], est de fournir des données aux informaticiens, sur lesquelles ils puissent exercer leurs talents. En ces temps difficiles où même de talentueux informaticiens sont au chômage, ce n'est pas négligeable. En revanche, pour ce qui est de la biologie fondamentale ou de la santé publique, l'intérêt du programme "Génome humain " est difficile à cerner.

Sur le plan fondamental, tout processus biologique se déroule dans le temps. On ne peut comprendre et maîtriser un quelconque phénomène biologique en faisant abstraction de la dimension temporelle, laquelle, jusqu'à nouvel ordre, est illisible dans le génome. Pour ce qui est des applications, il faudrait les placer dans le cadre plus large de la surconsommation médicale. Ne se porterait-on pas mieux si l'on dépensait moins à se faire examiner le nombril, et plus à se financer de saines vacances [2] ?

Au jeu d'échecs, quand un joueur dispose d'un léger avantage matériel disons qu'il a deux pions d'avance il peut, par une méthode presque infaillible, battre son adversaire. Il lui suffit de forcer les échanges: dame contre dame, tour contre tour, etc. Le rapport des forces évolue inexorablement en sa faveur. En fin de partie, un petit pion surnuméraire, amené sur la dernière ligne adverse se transforme en dame et fait mat.

Transposons au domaine scientifique. Soit un pays dominateur qui disposerait d'une science riche, diversifiée, et plus avancée dans bien des domaines que celle des autres pays du globe. Comment s'assurer un ascendant durable et décisif sur le reste du monde ? Rien de plus facile. On lance une grande action sur un thème numéro 1 , en proposant au reste du monde de s'y associer. Tombant dans le panneau, les stratèges scientifiques des autres pays proclament qu'il faut " ne pas se laisser distancer " par le grand frère, et lancent des actions sur le même thème. Un partout. Puis, le grand frère fait la promotion d'un grand thème mondial numéro 2. Les stratèges des autres pays clament alors qu'il faut " combler le retard ". Deux partout, et ainsi de suite, jusqu'à épuisement des petits pays. Le grand, lui, est assez riche pour entretenir, en sus de cette science de façade, de vraies bonnes petites équipes de recherche, qui feront de vraies découvertes, comme le pion qui devient dame aux échecs, en fin de partie. Bravo Tom Cech ! John Cairns et Barry Hall, chapeau!

Et nous, Français, n'avons-nous vraiment rien de mieux à faire en science que " combler des retards ", c'est-à-dire encourager systématiquement les retardataires? Ne vaudrait-il pas mieux soutenir ceux qui n'ont personne devant?

\section{Jacques Ninio}

Institu Jacques-Monod, 2, place Jussier, tour 43, 75251 Paris Cedex 05, France et École Normale Supérinue, 24, nue Lhomond 75231 Panis Cedex 05, France.

\section{RÉFÉRENCES}

1. Gilbert W. Towards a paradigm shift in biology. Nature 1991; 349 : 99.

2. Ninio J. La biologie buissonnière. Paris, Seuil, 1991.

- Collège de fRANCE • CHAIRE DE COMMUNICATIONS CELLULAIRES

Jean-Pierre Changeux, professeur Année 1991-1992

Cours : le lundi à $9 \mathrm{~h} 30$, salle 6

Bases neurales des états de conscience et d'attention

Séminaires : le lundi à $11 \mathrm{~h}$, salle 6 Imagerie cérébrale

6 avril : G. Charpak (CERN, Genève) Nouvelles méthodes d'imagerie cérébrale inspirées des techniques de détection de la physique des particules

13 avril : B. Mazoyer (CEA, Hôpital Frédéric-Joliot, Orsay)

Imagerie du langage par la tomographie à émission de positron

27 avril : A. Leroy-Willig (CEA, Hôpital Frédéric-Joliot, Orsay)

Imagerie fonctionnelle du cerveau par Résonance Magnétique

11 mai : A. Berthoz (Laboratoire de Physiologie Neurosensorielle, CNRS UPR 2, Paris)

Exploration des mécanismes de contrôle du mouvement par imagerie cérébrale

18 mai : C. Sotelo (INSERM U.106, La Salpêtrière, Paris)

Révélation de l'activité neuronale par I'histologie

25 mai : B. Renault (CNRS URA 654, La Salpêtrière, Paris)

La magnétoencéphalographie (Meg) : principe, limites et applications

1or juin : P. Chauvel (Clinique Neurologique, CHU Pontchaillou, Rennes)

Imagerie cérébrale par électroencéphalographie

L'Administrateur du Collège de France André Miquel 